\title{
Gravitomagnetism and Gravitational Waves
}

\author{
L. Iorio ${ }^{*}, 1$ and C. Corda ${ }^{*}, 2$ \\ ${ }^{I}$ Viale Unità di Italia 68, 70125, Bari (BA), Italy \\ ${ }^{2}$ Institute for Basic Research, P. O. Box 1577, Palm Harbor, FL 34682, USA
}

\begin{abstract}
After extensively reviewing general relativistic gravitomagnetism, both historically and phenomenologically, we review in detail the so-called magnetic components of gravitational waves (GWs), which have to be taken into account in the context of the total response functions of interferometers for GWs propagating from arbitrary directions. Following the more recent approaches of this important issue, the analysis of such magnetic components will be reviewed in both of standard General Theory of Relativity (GTR) and Scalar Tensor Gravity. Thus, we show in detail that such a magnetic component becomes particularly important in the high-frequency portion of the range of ground based interferometers for GWs which arises from the two different theories of gravity. Our reviewed results show that if one neglects the magnetic contribution to the gravitational field of a $\mathrm{GW}$, approximately $15 \%$ of the potential observable signal could, in principle, be lost.
\end{abstract}

Key Words: Experimental studies of gravity, Experimental tests of gravitational theories, Gravitational waves.

\section{GENERAL OVERVIEW OF GRAVITO- MAGNETISM}

The term "gravitomagnetism" [1-3] (GM) commonly indicates the collection of those gravitational phenomena regarding orbiting test particles, precessing gyroscopes, moving clocks and atoms and propagating electromagnetic waves [4,5] which, in the framework of the Einstein's General Theory of Relativity [6] (GTR), arise from nonstatic distributions of matter and energy. In the weak-field and slow motion approximation, the Einstein field equations [7] of GTR, which is a highly non-linear Lorentz-covariant tensor theory of gravitation, get linearized [8], thus looking like the Maxwellian equations of electromagntism [9]. As a consequence, a "gravitomagnetic" field $\vec{B}_{g}$, induced by the off-diagonal components $g_{0 i}, i=1,2,3$ of the space-time metric tensor related to mass-energy currents, arises. In particular, far from a localized rotating body with angular momentum $\vec{S}$ the gravitomagnetic field can be written as [10]

$$
\vec{B}_{g}(\vec{r})=\frac{G}{c r^{3}}[\vec{S}-3(\vec{S} \cdot \hat{r}) \hat{r}],
$$

where $G$ is the Newtonian gravitational constant and $c$ is the speed of light in vacuum. It affects, e.g., a test particle moving with velocity $\vec{v}$ with a non-central acceleration [10]

$$
\vec{A}_{\mathrm{G} M}=\left(\frac{\vec{v}}{c}\right) \times \vec{B}_{g},
$$

which is the cause of two of the most famous and empirically investigated GM effects, as we will see in Section 1.1 and Section 1.2.

*Address correspondence to this authors Viale Unità di Italia 68, 70125, Bari (BA), Italy; Tel: 0039328 6128815; Institute for Basic Research, P. O. Box 1577, Palm Harbor, FL 34682, USA; Tel: 003938034160 37;

E-mails: lorenzo.iorio@libero.it, cordac.galilei@gmail.com

\subsection{Historical Overview}

The formal analogies between gravitation and electromagnetism date back to the early days of the Coulomb's force law [11-13] between two non-moving pointlike electric charges (1785). Indeed, it follows faithfully the Newtonian inverse-square force law of gravitation [14] between two pointlike masses (1687) whose state of motion is, instead, irrelevant for its validity within the framework of classical mechanics. After the electrodynamical forces between current elements were discovered in 1820-1825 by Ampère [15], the situation was reversed. Indeed, in 1870 Holzmüller [16], in order to study the motion of a test particle attracted by a fixed center, asked whether Newton's law might not be modified in much the same way as that in which Weber $[17,18]$ in 1846 had modified Coulomb's law for electric charges in an action-at-a-distance fashion by introducing velocity-dependent terms. He found that the trajectory is no longer closed, but can be described by a slowly precessing ellipse. Later, Tisserand [19, 20], used this method, yielding a further, "magnetic" -like component of the Sun's gravitational field acting on the solar system's planets, to attempt-unsuccessfully-to explain the anomalous prograde perihelion precession of Mercury ${ }^{1}$ of 43.98 arcsec cty $^{-1}$, discovered in 1859 by Le Verrier [22]. Also Lévy [23] worked in the same conceptual framework to solve that astronomical problem, but without success. The advent of Maxwell's electromagnetism [24], which is a linear field theory replacing the previous action-at-a-distance theories ${ }^{2}$, did not discourage further attempts to use analogies of electromagnetic equations to solve gravitational problems.

${ }^{1}$ It found a natural explanation in 1915 by Einstein [21] within his GTR. Note that the static, "gravitoelectric" part of the Sun's field is required for the explanation of such a phenomenon: no mass-energy currents are involved.

${ }^{2} \mathrm{~A}$ recent action-at-a-distance gravity theory, based on the scalar velocitydependent Weber-type potential, is due to Assis [25]. 
Maxwell himself [24] considered whether Newtonian gravity could be described by a vector field theory, but he did not succeed because of issues encountered with gravitational energy. A later attempt was due to Heaviside [26] in 1894 with his Maxwellian vector field theory of gravity. Among other things, he derived a planetary precession induced by the rotating Sun's GM dipole, but it was too small in magnitude and retrograde with respect to the Mercury's precession observed by Le Verrier.

Another line of reasoning which yielded to consider gravitational forces induced by moving masses was that connected to the need of explaining the origin of inertia. In particular, the idea that rotating bodies may exert not only the static Newtonian gravitational force but also an additional "frame-dragging" on test particles was probably due to Mach [27]. He speculated that even for relative rotations centrifugal forces arise due to some, unspecified gravitational interaction with the masses of the Earth and of the other celestial bodies [28], certainly quite larger than the mass of the famous Newton's bucket in relative rotation with respect to the water inside. Later, in 1896 the Friedländer brothers [29] expressed the conviction that the properties of inertia and gravitation should be finally derived from a unified law. A Maxwell-like theory of gravitation, proposed to explain the origin of inertia in the framework of the Mach's principle, is due to Sciama [30].

After the birth of the Einstein's Special Theory of Relativity (STR) in 1905 [31], the problem of a "magnetic" -type component of the gravitational field of non-static mass distributions was tackled in the framework of the search for a consistent relativistic theory of gravitation [32]. Indeed, bringing together Newtonian gravitation and Lorentz invariance in a consistent field-theoretic framework necessarily requires the introduction of a "magnetic" -type gravitational field of some form [33-35].

With a preliminary and still incorrect version of GTR, Einstein and Besso in 1913 [36] calculated the node precession of planets in the field of the rotating Sun; the figures they obtained for Mercury and Venus were incorrect also because they used a wrong value for the solar mass. Soon after GTR was put forth by Einstein (1915) [37], de Sitter [38] in 1916 used it to preliminarily work out the effects of Sun's rotation on planets' perihelia, although he restricted himself to ecliptic orbits only; his result for Mercury $\left(-0.01 \operatorname{arcsec}^{-1}{ }^{-1}\right)$ was too large by one order of magnitude because he assumed a homogenous and uniformly rotating Sun. In 1918 Thirring [39] analyzed in a short article the formal analogies between the Maxwell equations and the linearized Einstein equations. Later [39-41], Thirring computed the centrifugal and Coriolis-like gravitomagnetic forces occurring inside a rotating massive shell. Lense and Thirring $^{3}$ [44] in 1918 worked out the gravitomagnetic effects on the orbital motions of test particles outside a slowly rotating mass; in particular, they computed the

\footnotetext{
${ }^{3}$ However, in August 1917 Einstein [42] wrote to Thirring that he calculated the Coriolis-type field of the rotating Earth and Sun, and its influence on the orbital elements of planets (and moons). A detailed history of the formulation of the so-called Lense-Thirring effect has recently been outlined by Pfister [43]; according to him, it would be more fair to speak about an Einstein-Thirring-Lense effect.
}

gravitomagnetic rates of both the satellites of Mars (Phobos and Deimos), and of some of the moons of the giant gaseous planets. They found for the longitude of the ascending node $\Omega$ a prograde precession, while for the argument of pericenter $\omega$ a retrograde precession occurs

$$
\dot{\Omega}_{\mathrm{LT}}=\frac{2 G S}{c^{2} a^{3}\left(1-e^{2}\right)^{\frac{3}{2}}}, \dot{\omega}_{\mathrm{LT}}=-\frac{6 G S \cos I}{c^{2} a^{3}\left(1-e^{2}\right)^{\frac{3}{2}}},
$$

where $a, e, I$ are the semimajor axis, the eccentricity and the inclination of the test particle's orbital plane to the central body's equator, respectively.

Another well known general relativistic gravitomagnetic effect consists of the precession of a gyroscope moving in the field of a slowly rotating body. It was derived in 1959 by Pugh [45] and in 1960 by Schiff [46-48]. The possible Machian character of the Schiff effect was discussed by Rindler [49] and Bondi and Samuel [50].

Cosmological GM and Mach's principle have been recently treated by Schmid [51, 52].

Certain subtle issues concerning the gravitomagnetic effects inside a rotating massive shell were solved by Pfister and Braun in 1985 [53].

For seeming analogies between Maxwellian electromagnetism and the fully non-linear equations of GTR, see the works by Matte [54] and, more recently, by Costa and Herdeiro [55]. In this framework, Pascual-Sánchez [56] discussed the non-existence of a GM dynamo in the linearized, weak-field and slow-motion approximation of GTR. Tartaglia and Ruggiero [57] investigated the possible occurrence of a GM analog of the Meissner effect using the same approximation for GTR. The non-existence of such a phenomenon in gravitation and of other putative GM effects has been demonstrated by Pascual-Sánchez [58].

\subsection{Experimental/Observational Overview}

About empirical investigations of possible gravitational effects due to moving bodies, in 1896 I. Friedländer [29] was the first to perform an experiment concerning a putative gravitational influence of moving bodies. He used as a source a rapidly rotating heavy fly wheel and triedunsuccessfully-to detect its gravitational effects on a torsion balance mounted above the fly wheel, in line with its axis. Later, in 1904 Föppl [59] looked for possible Coriolis-like gravitational dragging effects induced on a gyroscope made of two heavy fly wheels by the whole rotating Earth as a source.

Moving to more recent epochs, soon after the dawn of the space age with the launch of Sputnik in 1957 it was proposed by Soviet scientists to directly test the general relativistic Lense-Thirring effect with artificial satellites orbiting the Earth. In particular, V.L. Ginzburg [60-62], proposed to use the perigee of a terrestrial spacecraft in highly elliptic orbit, while A.F. Bogorodskii [63] considered also the node. In 1959 Yilmaz [64], aware of the aliasing effect of the much larger classical precessions induced by the non-sphericity of the Earth, proposed to launch a satellite in a polar orbit to cancel them. About twenty years later, in 1976 van Patten and Everitt [65, 66], suggested to use a pair of drag-free, counter-orbiting terrestrial spacecraft in nearly polar orbits to 
detect their combined Lense-Thirring node precessions. In 1977-1978 Cugusi and Proverbio [67, 68], suggested to use the passive geodetic satellite LAGEOS, in orbit around the Earth since 1976 and tracked with the Satellite Laser Ranging (SLR) technique, along with the other existing laser-ranged targets to measure the Lense-Thirring node precession. In 1986 Ciufolini [69] proposed a somewhat simpler version of the van Patten-Everitt mission consisting of looking at the sum of the nodes of LAGEOS and of another SLR satellite to be launched in the same orbit, apart from the inclination which should be switched by $180 \mathrm{deg}$ in order to minimize the competing classical precessions due to the centrifugal oblateness of the Earth. Iorio [70] showed that such an orbital configuration would allow, in principle, to use the difference of the perigees as well. Tests have started to be effectively performed later by Ciufolini and coworkers [71] with the LAGEOS and LAGEOS II satellites ${ }^{4}$, according to a strategy by Ciufolini [72] involving the use of a suitable linear combination of the nodes $\Omega$ of both satellites and the perigee $\omega$ of LAGEOS II in order to remove the impact of the first two multipoles of the nonspherical gravitational potential of the Earth. Latest tests have been reported by Ciufolini and Pavlis [73, 74], Lucchesi [75] and Ries and coworkers [76] with only the nodes of both the satellites according to a combination of them explicitly proposed by Iorio [77]. The total uncertainty reached is still matter of debate [78-84] because of the lingering uncertainties in the Earth's multipoles and in how to evaluate their biasing impact; it may be as large as $\approx 20-30 \%$ according to conservative evaluations $[78,81$ $84]$, while more optimistic views $[73,74,76]$ point towards $10-15 \%$. Several authors [85-89] explored the possibility of using other currently orbiting SLR geodetic satellites in addition to LAGEOS and LAGEOS II. A new SLR geodetic satellite, named LARES, should be launched by the Italian Space Agency (ASI) in 2010 to improve the present-day accuracy of the Lense-Thirring tests by combining its node with those of the existing LAGEOS and LAGEOS II [90]. The claimed accuracy is $1 \%$ [91], but also in this case the realistic level of uncertainty may be quite larger [92-95] because of the relatively low orbit of LARES with respect to LAGEOS and LAGEOS II which should bring into play the systematic alias by several non-perfectly known Earth's multipoles.

In 2006 a preliminary test in the gravitational field of Mars with the Mars Global Surveyor (MGS) has been performed by Iorio [96, 97]. He interpreted certain features of the time series of the out-of-plane portion $N$ of the MGS orbit involving its node in terms of the Lense-Thirring effect. In particular, the average of the Root-Mean-Square (RMS) orbit-overlap differences of the out-of-plane component of the MGS path over 5 years agrees with the predicted average Lense-Thirring out-of-plane shift over the same time span within a few percent, while a linear fit to the complete $N$ time series for the entire MGS data set shows an agreement with the corresponding predicted Lense-Thirring signal at an about $40 \%$ level. A debate about the validity of such an interpretation arose [98], and it is still ongoing [99]. The

${ }^{4}$ LAGEOS II was launched in 1992, but its orbital configuration is different from that proposed in [69]. possibility of designing a dedicated mission to Mars has been recently considered by Iorio [100].

Iorio and Lainey [101] revisited the original proposal by Lense and Thirring [44] concerning the system of Jovian moons in view of recent advances in orbit determination of the four large Galilean satellites. The possibility of using fruitfully them seem still to be premature.

Concerning the Sun's GM field and the inner planets, the situation is nowadays more favorable than in the past [38, 68]. Indeed, the astronomer Pitjeva [102, 103], has recently fitted the full set of dynamical force models of the planetary motions of the EPM ephemerides to about one century of data of several types by estimating, among other things, corrections $\Delta \dot{\varpi}$ to the standard Newtonian/Einsteinian secular precessions of the longitude of the perihelia of all the rocky planets. In doing so she did not model the solar GM field, so that such corrections, by construction, are well suited to test the Lense-Thirring effect [104-106]. The magnitude of the predicted Lense-Thirring perihelion precessions, although one order of magnitude smaller than what argued in earlier studies [38,68], is about of the same order of magnitude of, or even larger than, the present-day uncertainty in the estimated $\Delta \dot{\boldsymbol{\varpi}}$. In particular, it has been noted [106] that the Lense-Thirring perihelion precession of Venus amounts to $\dot{\varpi}_{\mathrm{LT}}=-0.0003 \operatorname{arcsec} \mathrm{cty}^{-1}$, while the estimated correction for Venus is $\Delta \dot{\varpi}=-0.0004 \pm 0.0001$, where the quoted uncertainty is the $1-\sigma$ statistical error. Thus, the existence of the Lense-Thirring effect would be confirmed at a $25 \%$ level, although caution is in order because the realistic uncertainty in $\Delta \dot{\varpi}$ might be up to 5 times larger. The systematic bias due to the mismodeling of other competing classical effects would be less relevant than in the LAGEOS-LAGEOS II case. The proposed spacebased Astrodynamical Space Test of Relativity using Optical Devices (ASTROD) mission [107] has, among its scientific goals, also the accurate determination of the Sun's angular momentum through a $\approx 14 \%$ measurement of the gravitomagnetic time delay on electromagnetic waves.

Soon after the formulation of the Schiff effect, in 1961 Fairbank and Schiff [108] submitted to NASA a proposal for a dedicated space-based experiment aimed to directly measure it. Such an extremely complicated mission, later named Gravity Probe B (GP-B) [109, 110], consisted of a drag-free, liquid helium-cooled spacecraft moving in a polar, low orbit around the Earth and carrying onboard four superconducting gyroscopes whose GM precessions should have been detected by Superconducting Quantum Interference Devices (SQUID) with an expected accuracy of $1 \%$ or better. It took 43 years to be implemented since GPB was finally launched on 20 April 2004; the science data collection lasted from 27 August 2004 to 29 September 2005 , while the data analysis is still ongoing [111, 112]. It seems that the final accuracy obtainable will be not so good as initially hoped because of the occurrence of unexpected systematic errors [113-115]. At present, GP-B team reports ${ }^{5}$ a statistical error of approximately $14 \%$ and systematic uncertainty of $\approx 10 \%$. In 1975 Haas and Ross [116] proposed

\footnotetext{
${ }^{5}$ See on the WEB: http://einstein.stanford.edu/
} 
to measure the angular momenta of the Sun and Jupiter by exploiting the Schiff effect with dedicated spacecraft-based missions, but such a proposal was not carried out so far.

All the previously reviewed attempts aim to obtain direct tests of some GM effects. However, according to Nordtvedt $[117,118]$, the GM interaction would have already been observed, with a relative accuracy of 1 part to 1000 , in comprehensive fits of the motions of several astronomical and astrophysical bodies like satellites, binary pulsars and the Moon. In fact, Nordtvedt does not refer to the effects considered so far, caused by the rotation of the body which acts as source of the gravitational field ("intrinsic" GM). Instead, he primarily deals with some GM long-periodic harmonic perturbations affecting the Earth-Moon range induced by the translational GM mass currents due to the orbital motion of the Earth-Moon system around the Sun ("extrinsic" GM); Lunar Laser Ranging (LLR) would have measured them with a $0.1 \%$ accuracy [119]. However, Kopeikin argues that LLR would not be able to detect genuine GM signatures which are not spurious, gaugedependent effects [120]. For other works about such an issue, see [121-123]. Concerning the possibility of directly measuring the Lense-Thirring precessions of the Moon's motion due to the Earth's angular momentum, it has been recently proven to be still unfeasible by Iorio [124] because of the too large level of uncertainty in several competing classical effects. A test of extrinsic gravitomagn-etism concerning the deflection of electromagnetic waves by Jupiter in its orbital motion has been reported in a dedicated analysis of radiointerferometric data by Fomalont and Kopeikin [125], but also such a test is controversial: see the WEB page by Will at http://physics.wustl.edu/cmw/Speedof Gravity.html.

For other proposals to directly detect various aspects of the (intrinsic) GM field in Earth-based laboratory and spacebased experiments, see, e.g., the book by Iorio [126]. Extensive overviews of the importance of GM in astrophysical contexts like accretion disks around compact objects and relativistic jets in quasars and galactic nuclei can be found, e.g., in the book by Thorne, Price and MacDonald [127], and in Section $E$ of the book by Ruffini and Sigismondi [128], and in the recent review article [129].

\section{MAGNETIC COMPONENT IN THE GRAVITATIONAL FIELD OF A GRAVITATIONAL WAVE}

\subsection{The Importance of Gravitational Waves: A New Window into the Universe}

The data analysis of interferometric Gravitational Waves (GWs) detectors has nowadays been started, and the scientific community hopes in a first direct detection of GWs in next years; for the current status of GWs interferometers see Ref. [130]. In such a way, the indirect evidence of the existence of GWs by Hulse and Taylor [131], Nobel Prize winners, will be confirmed. Detectors for GWs will be important for a better knowledge of the Universe [130] and also because the interferometric GWs detection will be the definitive test for GTR or, alternatively, a strong endorsement for Extended Theories of Gravity [132]. In fact, if advanced projects on the detection of GWs improve their sensitivity, allowing the Scientific Community to perform a
GW astronomy, accurate angle- and frequency-dependent response functions of interferometers for GWs arising from various theories of gravity will permit to discriminate among GTR and extended theories of gravity. This ultimate test will work because standard GTR admits only two polarizations for GWs, while in all extended theories the polarizations are, at least, three, see [132] for details.

On the other hand, the discovery of GW emission by the compact binary system composed by two Neutron Stars PSR1913+16 [131] has been, for physicists working in this field, the ultimate thrust allowing to reach the extremely sophisticated technology needed for investigating in this field of research [130].

Gravitational Waves are a consequence of Einstein's GTR [133], which presuppose GWs to be ripples in the space-time curvature travelling at light speed [134, 135]. Only asymmetric astrophysics sources can emit GWs. The most efficient are coalescing binaries systems, while a single rotating pulsar can rely only on spherical asymmetries, usually very small. Supernovae could have relevant asymmetries, being potential sources [130].

The most important cosmological source of GWs is, in principle, the so called stochastic background of GWs which, together with the Cosmic Background Radiation (CBR), would carry, if detected, a huge amount of information on the early stages of the Universe evolution [136-138, 143]. The existence of a relic stochastic background of GWs is a consequence of generals assumptions. Essentially it derives from a mixing between basic principles of classical theories of gravity and of quantum field theory. The strong variations of the gravitational field in the early universe amplify the zero-point quantum oscillations and produce relic GWs. It is well known that the detection of relic GWs is the only way to learn about the evolution of the very early universe, up to the bounds of the Planck epoch and the initial singularity $[139,140]$. It is very important to stress the unavoidable and fundamental character of this mechanism. The model derives from the inflationary scenario for the early universe [141], which is tuned in a good way with the WMAP data on the CBR (in particular exponential inflation and spectral index $\approx 1$ [142]). Inflationary models are cosmological models in which the Universe undergoes a brief phase of a very rapid expansion in early times [141]. In this context the expansion could be power-law or exponential in time. Such models provide solutions to the horizon and flatness problems and contain a mechanism which creates perturbations in all fields [139-141]. Important for our case is that this mechanism also provides a distinctive spectrum of relic GWs [139, 140]. The GWs perturbations arise from the uncertainty principle and the spectrum of relic GWs is generated from the adiabatically-amplified zero-point fluctuations $[139,140]$.

Regarding the potential GW detection, let us recall some historical notes.

In 1957, F.A.E. Pirani, who was a member of the Bondi's research group, proposed the geodesic deviation equation as a tool for designing a practical GW detector [144].

In 1959, Joseph Weber studied a detector that, in principle, might be able to measure displacements smaller than the size of the nucleus [145]. He developed an experiment using a large suspended bar of aluminum, with a 
high resonant $\mathrm{Q}$ at a frequency of about $1 \mathrm{kHz}$. Then, in 1960, he tried to test the general relativistic prediction of gravitational waves from strong gravity collisions [146] and, in 1969, he claimed evidence for observation of gravitational waves (based on coincident signals) from two bars separated by $1000 \mathrm{~km}$ [147]. He also proposed the idea of doing an experiment to detect gravitational waves using laser interferometers [147]. In fact, all the modern detectors can be considered like being originated from early Weber's ideas [130].

At the present time, in the world there are five cryogenic bar detectors which have been built to work at very low temperatures $(<4 K)$ : Explorer at CERN, Nautilus at Frascati INFN National Laboratory, Auriga at Legnaro National Laboratory, Allegro at Luisiana State University and Niobe in Perth [130]. Instrumental details can be found in [130] and references within. Spherical detectors are the Mario Schenberg, which has been built in San Paolo (Brazil) and the MiniGRAIL, which has been built at the Kamerlingh Onnes Laboratory of Leiden University, see [130] and references within. Spherical detectors are important for the potential detection of the scalar component of GWs that is admitted by Extended Theories of Gravity [148]. In the case of interferometric detectors, free falling masses are interferometer mirrors which can be separated by kilometres ( $3 \mathrm{~km}$ for Virgo, $4 \mathrm{~km}$ for LIGO). In this way, GW tidal force is, in principle, several order of magnitude larger than in bar detectors. Interferometers have very large bandwidth (10$10000 \mathrm{~Hz}$ ) because mirrors are suspended to pendulums having resonance in the $\mathrm{Hz}$ region. Thus, above such a resonance frequency, mirrors works, in a good approximation, like freely falling masses in the horizontal plane [130].

Recently, starting from the analysis in Ref. [149], some papers in literature have shown the importance of the gravitomagnetic effects in the framework of the GWs detection too $[151,152]$. In fact, the so-called magnetic components of GWs have to be taken into account in the context of the total response functions of interferometers for GWs propagating from arbitrary directions [149-152]. In next analysis we will show that such a magnetic component becomes particularly important in the high-frequency portion of the range of ground based interferometers for GWs which arises from standard GTR.

In a recent paper, the magnetic component has been extended to GWs arising from scalar-tensor gravity too [153]. In particular, in Ref. [153] it has been shown that if one neglects the magnetic contribution considering only the low-frequency approximation of the electric contribution, a portion of about the $15 \%$ of the signal could be, in principle, lost in the case of Scalar Tensor Gravity too, in total analogy with the standard case of GTR [149-152].

For the sake of completeness, such a case will be included in the following discussion.

\subsection{The magnetic Component of GWs in Standard GTR}

In a laboratory environment on Earth, coordinate systems in which the space-time is locally flat are typically used, and the distance between any two points is given simply by the difference in their coordinates in the sense of Newtonian physics [153-155]. In this frame, called the frame of the local observer, GWs manifest them-self by exerting tidal forces on the masses (the mirror and the beam-splitter in the case of an interferometer [150-153], see Fig. (1).

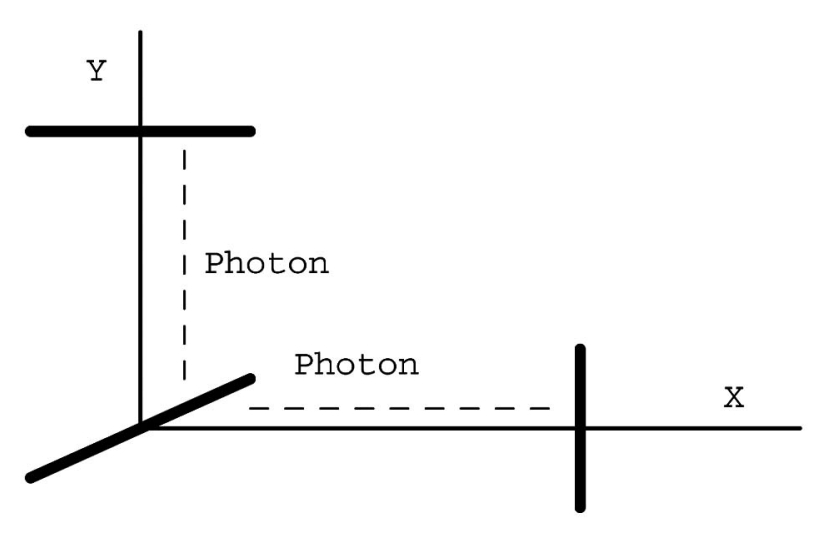

Fig. (1). photons can be launched from the beam-splitter to be bounced back by the mirror.

The presence and importance of the so-called magnetic components of GWs in the framework of GTR was emphasized by Baskaran and Grishchuk that computed the correspondent detector patterns [149], while more detailed angular and frequency dependences of the response functions for the magnetic components have been given, with a specific application to the parameters of the LIGO and Virgo interferometers, in Refs. [150-152]. Thus, following Refs. [150-152], we will, now, work with $G=1, c=1$ and $\hbar=1$ and can call $h_{+}\left(t_{t t}+z_{t t}\right)$ and $h_{x}\left(t_{t t}+z_{t t}\right)$ the weak perturbations due to the + and the $\times$ polarizations which are expressed in terms of synchronous coordinates $t_{t t}, x_{t t}, y_{t t}, z_{t t}$ in the transverse-traceless (TT) gauge. In this way, the most general GW propagating in the $z_{t t}$ direction can be written in terms of a plane monochromatic wave [150-152]

$$
\begin{aligned}
& h_{\mu v}\left(t_{t t}+z_{t t}\right)=h_{+}\left(t_{t t}+z_{t t}\right) e_{\mu v}^{(+)}+h_{\times}\left(t_{t t}+z_{t t}\right) e_{\mu v}^{(\times)}= \\
& =h_{+0} \exp i \omega\left(t_{t t}+z_{t t}\right) e_{\mu v}^{(+)}+h_{\times 0} \exp i \omega\left(t_{t t}+z_{t t}\right) e_{\mu \nu}^{(\times)},
\end{aligned}
$$

and the corresponding line element will be

$$
d s^{2}=d t_{t t}^{2}-d z_{t t}^{2}-\left(1+h_{+}\right) d x_{t t}^{2}-\left(1-h_{+}\right) d y_{t t}^{2}-2 h_{\times} d x_{t t} d x_{t t}
$$

The wordlines $x_{t t}, y_{t t}, z_{t t}=$ const . are timelike geodesics representing the histories of free test masses [149-152]. The coordinate transformation $x^{\alpha}=x^{\alpha}\left(x_{t t}^{\beta}\right)$ from the TT coordinates to the frame of the local observer is [149-152] 


$$
\begin{gathered}
t=t_{t t}+\frac{1}{4}\left(x_{t t}^{2}-y_{t t}^{2}\right) \dot{h}_{+}-\frac{1}{2} x_{t t} y_{t t} \dot{h}_{\times} \\
x=x_{t t}+\frac{1}{2} x_{t t} h_{+}-\frac{1}{2} y_{t t} h_{\times}+\frac{1}{2} x_{t t} z_{t t} \dot{h}_{+}-\frac{1}{2} y_{t t} z_{t t} \dot{h}_{\times} \\
y=y_{t t}+\frac{1}{2} y_{t t} h_{+}-\frac{1}{2} x_{t t} h_{\times}+\frac{1}{2} y_{t t} z_{t t} \dot{h}_{+}-\frac{1}{2} x_{t t} z_{t t} \dot{h}_{\times} \\
z=z_{t t}-\frac{1}{4}\left(x_{t t}^{2}-y_{t t}^{2}\right) \dot{h}_{+}+\frac{1}{2} x_{t t} y_{t t} \dot{h}_{\times},
\end{gathered}
$$

where it is $\dot{h}_{+} \equiv \frac{\partial h_{+}}{\partial t}$ and $\dot{h}_{\times} \equiv \frac{\partial h_{\times}}{\partial t}$. The coefficients of this transformation (components of the metric and its first time derivative) are taken along the central wordline of the local observer [149-152]. It is well known from Refs. [149-152] that the linear and quadratic terms, as powers of $x_{t t}^{\alpha}$, are unambiguously determined by the conditions of the frame of the local observer, while the cubic and higher-order corrections are not determined by these conditions. Thus, at high-frequencies, the expansion in terms of higher-order corrections breaks down [149-152].

Considering a free mass riding on a timelike geodesic ( $x=l_{1}, y=l_{2}, \quad z=l_{3}$ ) [149-152], eq. (6) defines the motion of this mass with respect to the introduced frame of the local observer. In concrete terms one gets

$$
\begin{gathered}
x(t)=l_{1}+\frac{1}{2}\left[l_{1} h_{+}(t)-l_{2} h_{\times}(t)\right]+\frac{1}{2} l_{1} l_{3} \dot{h}_{+}(t)+\frac{1}{2} l_{2} l_{3} \dot{h}_{\times}(t) \\
y(t)=l_{2}-\frac{1}{2}\left[l_{2} h_{+}(t)+l_{1} h_{\times}(t)\right]-\frac{1}{2} l_{2} l_{3} \dot{h}_{+}(t)+\frac{1}{2} l_{1} l_{3} \dot{h}_{\times}(t) \\
z(t)=l_{3}-\frac{1}{4}\left(l_{1}^{2}-l_{2}^{2}\right) \dot{h}_{+}(t)+2 l_{1} l_{2} \dot{h}_{\times}(t),
\end{gathered}
$$

which are exactly eqs. (13) of Ref. [149-152] rewritten using the notation of Refs. [150-152]. In absence of GWs, the position of the mass is $\left(l_{1}, l_{2}, l_{3}\right)$. The effect of the $\mathrm{GW}$ is to drive the mass to have oscillations. Thus, in general, from eq. (7) all three components of motion are present [149-152]. Neglecting the terms with $\dot{h}_{+}$and $\dot{h}_{\times}$in eq. (7), the traditional equations for the mass motion are obtained [149152]

$$
\begin{gathered}
x(t)=l_{1}+\frac{1}{2}\left[l_{1} h_{+}(t)-l_{2} h_{\times}(t)\right] \\
y(t)=l_{2}-\frac{1}{2}\left[l_{2} h_{+}(t)+l_{1} h_{\times}(t)\right] \\
z(t)=l_{3} .
\end{gathered}
$$

Clearly, this is the analogous of the electric component of motion in electrodynamics [150-152], while equations

$$
\begin{gathered}
x(t)=l_{1}+\frac{1}{2} l_{1} l_{3} \dot{h}_{+}(t)+\frac{1}{2} l_{2} l_{3} \dot{h}_{\times}(t) \\
y(t)=l_{2}-\frac{1}{2} l_{2} l_{3} \dot{h}_{+}(t)+\frac{1}{2} l_{1} l_{3} \dot{h}_{\times}(t) \\
z(t)=l_{3}-\frac{1}{4}\left(l_{1}^{2}-l_{2}^{2}\right) \dot{h}_{+}(t)+2 l_{1} l_{2} \dot{h}_{\times}(t),
\end{gathered}
$$

are the analogous of the magnetic component of motion. One could think that the presence of these magnetic components is a frame artefact due to the transformation eq. (6), but in Section 4 of Ref. [149] eq. (7) have been directly obtained from the geodesic deviation equation too, thus the magnetic components have a real physical significance. The fundamental point of Ref. [149-152] is that the magnetic components become important when the frequency of the wave increases but only in the low-frequency regime. This can be understood directly from eq. (7). In fact, using eq. (4) and eq. (6), eq. (7) become

$$
\begin{gathered}
x(t)=l_{1}+\frac{1}{2}\left[l_{1} h_{+}(t)-l_{2} h_{\times}(t)\right]+\frac{1}{2} l_{1} l_{3} \omega h_{+}\left(t-\frac{\pi}{2}\right)+\frac{1}{2} l_{2} l_{3} \omega h_{\times}\left(t-\frac{\pi}{2}\right) \\
y(t)=l_{2}-\frac{1}{2}\left[l_{2} h_{+}(t)+l_{1} h_{\times}(t)\right]-\frac{1}{2} l_{2} l_{3} \omega h_{+}\left(t-\frac{\pi}{2}\right)+\frac{1}{2} l_{1} l_{3} \omega h_{\times}\left(t-\frac{\pi}{2}\right)(10 \\
z(t)=l_{3}-\frac{1}{4}\left(l_{1}^{2}-l_{2}^{2}\right) \omega h_{+}\left(t-\frac{\pi}{2}\right)+2 l_{1} l_{2} \omega h_{\times}\left(t-\frac{\pi}{2}\right) .
\end{gathered}
$$

Thus, the terms with $\dot{h}_{+}$and $\dot{h}_{x}$ in eq. (7) can be neglected only when the wavelength goes to infinity [149152], while, at high-frequencies, the expansion in terms of $\omega l_{i} l_{j}$ corrections, with $i, j=1,2,3$, breaks down [149-152].

Now, let us compute the total response functions of interferometers for the magnetic components in standard GTR.

Equations eq. (7), that represent the coordinates of the mirror of the interferometer in presence of a $\mathrm{GW}$ in the frame of the local observer, can be rewritten for the pure magnetic component of the + polarization as

$$
\begin{gathered}
x(t)=l_{1}+\frac{1}{2} l_{1} l_{3} \dot{h}_{+}(t) \\
y(t)=l_{2}-\frac{1}{2} l_{2} l_{3} \dot{h}_{+}(t) \\
z(t)=l_{3}-\frac{1}{4}\left(l_{1}^{2}-l_{2}^{2}\right) \dot{h}_{+}(t),
\end{gathered}
$$

where $l_{1}, l_{2}$ and $l_{3}$ are the unperturbed coordinates of the mirror.

To compute the response functions for an arbitrary propagating direction of the $\mathrm{GW}$, one recalls that the arms of 
the interferometer are in general in the $u$ and $v$ directions, while the $x, y, z$ frame is adapted to the propagating $\mathrm{GW}$ (i.e. the observer is assumed located in the position of the beam splitter). Then, a spatial rotation of the coordinate system has to be performed:

$$
\begin{array}{cc}
u= & -x \cos \theta \cos \phi+y \sin \phi+z \sin \theta \cos \phi \\
v= & -x \cos \theta \sin \phi-y \cos \phi+z \sin \theta \sin \phi \\
w= & x \sin \theta+z \cos \theta,
\end{array}
$$

or, in terms of the $x, y, z$ frame:

$$
\begin{array}{ll}
x= & -u \cos \theta \cos \phi-v \cos \theta \sin \phi+w \sin \theta \\
y= & u \sin \phi-v \cos \phi \\
z= & u \sin \theta \cos \phi+v \sin \theta \sin \phi+w \cos \theta .
\end{array}
$$

In this way, the GW is propagating from an arbitrary direction $r$ to the interferometer (see Fig. 2).

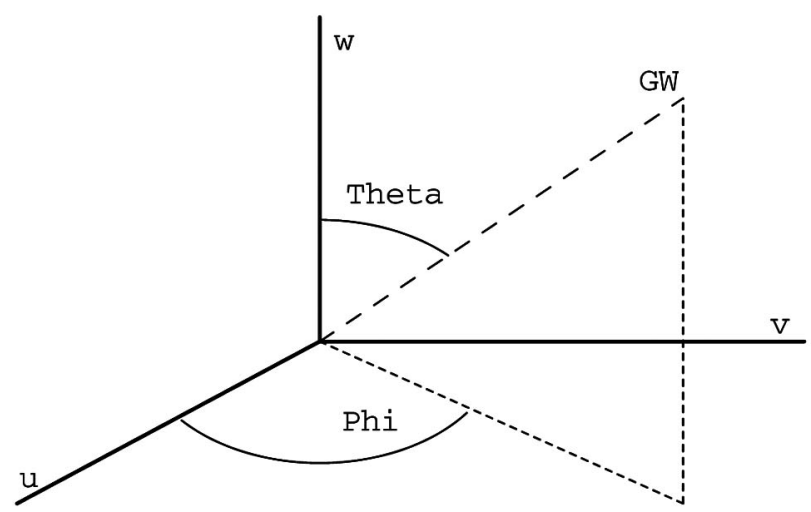

Fig. (2). a GW propagating from an arbitrary direction.

As the mirror of eq. (11) is situated in the $u$ direction, using eq. (11), eq. (12) and eq. (13) the $u$ coordinate of the mirror is given by

$$
u=L+\frac{1}{4} L^{2} A \dot{h}_{+}(t)
$$

where

$$
A=\sin \theta \cos \phi\left(\cos ^{2} \theta \cos ^{2} \phi-\sin ^{2} \phi\right)
$$

and $L=\sqrt{l_{1}^{2}+l_{2}^{2}+l_{3}^{2}}$ is the length of the interferometer arms.

The computation for the $v$ arm is similar to the one above. Using eq. (11), eq. (12) and eq. (13), the coordinate of the mirror in the $v$ arm is

$$
v=L+\frac{1}{4} L^{2} B \dot{h}_{+}(t),
$$

where

$$
B=\sin \theta \sin \phi\left(\cos ^{2} \theta \cos ^{2} \phi-\sin ^{2} \phi\right) .
$$

Eq. (14) and eq. (16) represent the distance of the two mirrors of the interferometer from the beam-splitter in presence of the GW (note that only the contribution of the magnetic component of the + polarization of the $\mathrm{GW}$ is taken into account). They represent particular cases of the more general form given in eq. (33) of [149].

A signal can also be defined in the time domain ( $T=L$ in our notation)

$$
\frac{\delta T(t)}{T}=\frac{u-v}{L}=\frac{1}{4} L(A-B) \dot{h}_{+}(t) .
$$

The quantity eq. (18) can be computed in the frequency domain by using the Fourier transform of $h_{+}$, defined by

$$
\tilde{h}_{+}(\omega)=\int_{-\infty}^{\infty} d t h_{+}(t) \exp (i \omega t),
$$

obtaining

$$
\frac{\tilde{\delta} T(\omega)}{T}=H_{\mathrm{m} a g n}^{+}(\omega) \tilde{h}_{+}(\omega),
$$

where the function

$$
\begin{gathered}
H_{\mathrm{m} a g n}^{+}(\omega)=-\frac{1}{8} i \omega L(A-B)= \\
=-\frac{1}{4} i \omega L \sin \theta\left[\left(\cos ^{2} \theta+\sin ^{2} \phi \frac{1+\cos ^{2} \theta}{2}\right)\right](\cos \phi-\sin \phi)
\end{gathered}
$$

is the total response function of the interferometer for the magnetic component of the + polarization [149-152].

In the above computation the theorem on the derivative of the Fourier transform has been used.

The angular dependence of the response function eq. (20) of the LIGO interferometer to the magnetic component of the + polarization for $f=8000 \mathrm{~Hz}$ is shown in Fig. (3).

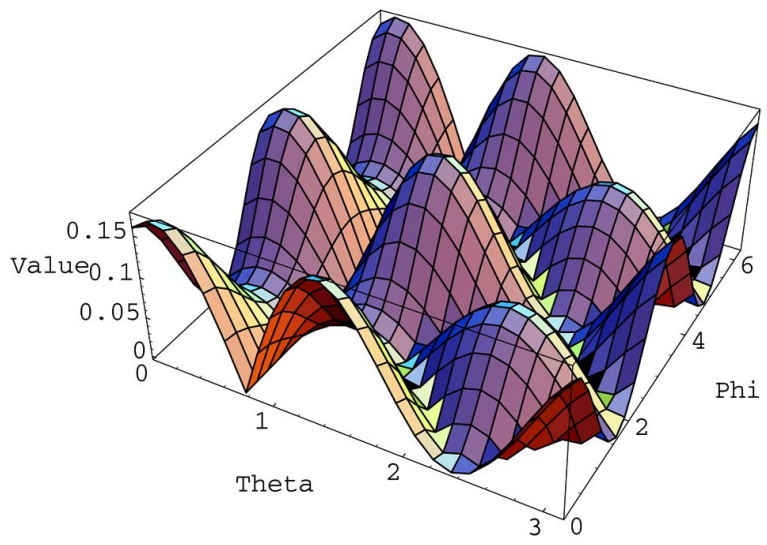

Fig. (3). the angular dependence of the response function of the LIGO interferometer to the magnetic component of the + polarization for $f=8000 \mathrm{~Hz}$.

The analysis can be generalized for the magnetic component of the $\times$ polarization too. In this case, eq. (7) can be rewritten for the pure magnetic component of the $\times$ polarization as [149-152] 


$$
\begin{aligned}
& x(t)=l_{1}+\frac{1}{2} l_{2} l_{3} \dot{h}_{\times}(t) \\
& y(t)=l_{2}+\frac{1}{2} l_{1} l_{3} \dot{h}_{\times}(t) \\
& z(t)=l_{3}-\frac{1}{2} l_{1} l_{2} \dot{h}_{\times}(t) .
\end{aligned}
$$

Using eq. (21), eq. (12) and eq. (13), the $u$ coordinate of the mirror in the $u$ arm of the interferometer is given by

$$
u=L+\frac{1}{4} L^{2} C \dot{h}_{\times}(t),
$$

where

$$
C \equiv-2 \cos \theta \cos ^{2} \phi \sin \theta \sin \phi
$$

while the $v$ coordinate of the mirror in the $v$ arm of the interferometer is given by

$$
v=L+\frac{1}{4} L^{2} D \dot{h}_{\times}(t),
$$

with

$$
D \equiv 2 \cos \theta \cos \phi \sin \theta \sin ^{2} \phi .
$$

Thus, with an analysis similar to the one of previous Sections, it is possible to show that the response function of the interferometer for the magnetic component of the $x$ polarization is [149-152]

$$
\begin{gathered}
H_{\mathrm{m} a g n}^{\times}(\omega)=-i \omega T(C-D)= \\
=-i \omega L \sin 2 \phi(\cos \phi+\sin \phi) \cos \theta,
\end{gathered}
$$

The angular dependence of the response function eq. (26) of the LIGO interferometer to the magnetic component of the $\times$ polarization for $f=8000 \mathrm{~Hz}$ is shown in Fig. (4).

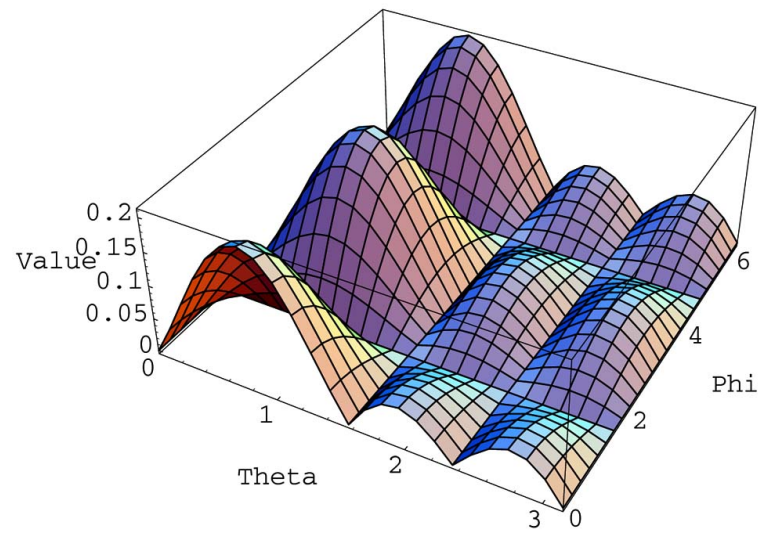

Fig. (4). The angular dependence of the total response function of the LIGO interferometer to the magnetic component of the $x$ polarization for $f=8000 \mathrm{~Hz}$.

From Fig. (3) and Fig. (4), it looks clear that if one neglects the magnetic contribution, approximately $15 \%$ of currently observable signal could, in principle, be lost.

\subsection{The Magnetic Component of GWs in Scalar Tensor Gravity}

In the framework of Scalar Tensor Gravity, the TT gauge can be extended to a third polarization [132, 153, 157-160]. In this way, the total perturbation of a gravitational wave propagating in the $z$ - direction in this gauge is $[132,153$, 156-160]

$$
h_{\mu v}(t+z)=A^{+}(t+z) e_{\mu \nu}^{(+)}+A^{\times}(t+z) e_{\mu \nu}^{(\times)}+\Phi(t+z) e_{\mu v}^{(s)}
$$

The term $A^{+}(t+z) e_{\mu \nu}^{(+)}+A^{\times}(t+z) e_{\mu \nu}^{(\times)}$describes the two standard (i.e. tensor) polarizations of gravitational waves which arises from General Relativity in the TT gauge, see previous Subsection, while the term $\Phi(t+z) e_{\mu v}^{(s)}$ is the extension of the TT gauge to the scalar-tensor case.

For a purely scalar GW the metric perturbation eq. (27) reduces to $[132,153,160]$

$$
h_{\mu v}=\Phi e_{\mu v}^{(s)}
$$

and the correspondent line element is $[132,153,160]$

$$
d s^{2}=d t^{2}-d z^{2}-(1+\Phi) d x^{2}-(1+\Phi) d y^{2}
$$

with $\Phi=\Phi_{0} e^{i \omega(t+z)}$.

Again, the wordlines $x, y, z=$ const. are timelike geodesics representing the histories of free test masses, see the analogy with tensor waves in previous Subsection. In this case, the coordinate transformation $x^{\alpha}=x^{\alpha}\left(x_{t t}^{\beta}\right)$ from the TT coordinates to the frame of the local observer is [153]

$$
\begin{gathered}
t=t_{t t}+\frac{1}{4}\left(x_{t t}^{2}-y_{t t}^{2}\right) \dot{\Phi} \\
x=x_{t t}+\frac{1}{2} x_{t t} \Phi+\frac{1}{2} x_{t t} z_{t t} \dot{\Phi} \\
y=y_{t t}+\frac{1}{2} y_{t t} \Phi+\frac{1}{2} y_{t t} z_{t t} \dot{\Phi} \\
z=z_{t t}-\frac{1}{4}\left(x_{t t}^{2}-y_{t t}^{2}\right) \dot{\Phi},
\end{gathered}
$$

where it is $\dot{\Phi} \equiv \frac{\partial \Phi}{\partial t}$, see previous Subsection and [153].

Now, if one considers a free mass riding on a timelike geodesic ( $x=l_{1}, y=l_{2}, z=l_{3}$ ), eq. (30) define the motion of this mass due to the scalar GW with respect to the introduced frame of the local observer. Thus, one gets 


$$
\begin{gathered}
x(t)=l_{1}+\frac{1}{2} l_{1} \Phi(t)+\frac{1}{2} l_{1} l_{3} \dot{\Phi}(t) \\
y(t)=l_{2}+\frac{1}{2} l_{2} \Phi(t)+\frac{1}{2} l_{2} l_{3} \dot{\Phi}(t) \\
z(t)=l_{3}-\frac{1}{4}\left(l_{1}^{2}-l_{2}^{2}\right) \dot{\Phi}(t) .
\end{gathered}
$$

In absence of scalar GWs the position of the mass is $\left(l_{1}, l_{2}, l_{3}\right)$. Again, the effect of the scalar $\mathrm{GW}$ is to drive the mass to have oscillations. Thus, in general, from eq. (31) all three components of motion are present.

Neglecting the terms with $\dot{\Phi}$ in eq. (31), the traditional equations for the mass motion due to the scalar GW are obtained [153]

$$
\begin{gathered}
x(t)=l_{1}+\frac{1}{2} l_{1} \Phi(t) \\
y(t)=l_{2}+\frac{1}{2} l_{2} \Phi(t) \\
z(t)=l_{3} .
\end{gathered}
$$

This is the analogous of the electric component of motion in electrodynamics (see previous Subsection), while equations

$$
\begin{gathered}
x(t)=l_{1}+\frac{1}{2} l_{1} l_{3} \dot{\Phi}(t) \\
y(t)=l_{2}+\frac{1}{2} l_{2} l_{3} \dot{\Phi}(t) \\
z(t)=l_{3}-\frac{1}{4}\left(l_{1}^{2}-l_{2}^{2}\right) \dot{\Phi}(t),
\end{gathered}
$$

are the analog of the magnetic component of motion.

Thus, the magnetic component becomes important when the frequency of the wave increases in this case too, but only in the low-frequency regime in analogy with the standard tensor case.

Even in this scalar case, one could think that the presence of this magnetic component is a frame artefact due to the transformation eq. (30), but now we show that eq. (33) can be directly obtained from the geodesic deviation equation too, proving that the magnetic components have a real physical significance.

Following [153], let us focus the attention on the geodesic deviation extended to second order approximation. The derivation of the geodesic deviation equations is usually based on a two parameter family of timelike geodesic $x^{\alpha}(\tau, r)$. In general and in the lowest approximation, the geodesic deviation equations are given by $[153,160]$

$$
\frac{D^{2} n^{\delta}}{d \tau^{2}}=R_{\alpha \beta \gamma}^{\delta} u^{\alpha} u^{\gamma} n^{\beta}
$$

The vector $u^{\alpha}$ is the unit tangent vector to the geodesic and $n^{\alpha}$ is the separation vector between two nearby geodesics

$$
u^{\alpha}(\tau, r)=\left.\frac{\partial x^{\alpha}}{\partial \tau}\right|_{r=\text { const. }}
$$

$$
n^{\alpha}(\tau, r)=\left.\frac{\partial x^{\alpha}}{\partial r}\right|_{t=\text { const. }} .
$$

It is also assumed that the central geodesic line corresponds to $r=0$ while the second nearby geodesic corresponds to $r=r_{0}$ [153]. Then, $R_{\alpha \beta \gamma}{ }^{\delta}$ in eq. (34) is the curvature tensor calculated along the central geodesic and $\frac{D}{d \tau}$ the covariant derivative calculated along that line [153].

To discuss the magnetic component of motion in the field of a scalar $\mathrm{GW}$, we need the geodesic deviation equations extended to the next approximation. These equations have been obtained in [153]. Let us introduce the closely related vector $w^{\alpha}$

$$
w^{\alpha}=\frac{D n^{\alpha}}{d r}=n_{; \beta}^{\alpha} n^{\beta}=\frac{\partial^{2} x^{\alpha}}{\partial r^{2}}+\Gamma_{\beta \gamma}^{\alpha} u^{\beta} u^{\gamma} .
$$

This vector obeys the equations [153]

$$
\frac{D^{2} w^{\delta}}{d \tau^{2}}=R_{\alpha \beta \gamma}^{\delta} u^{\alpha} u^{\gamma} w^{\beta}+\left(R_{\alpha \beta \gamma ; \varepsilon}^{\delta}-R_{c \varepsilon \alpha ; \beta}^{\delta}\right) u^{\alpha} u^{\beta} u^{\gamma} u^{\varepsilon}+4 R_{\alpha \beta \gamma}{ }^{\delta} u^{\beta} \frac{D n^{\alpha}}{d \tau} n^{\gamma} .
$$

Defining the vector

$$
N^{\alpha} \equiv r_{0} n^{\alpha}+\frac{1}{2} r_{0}^{2} w^{\alpha}
$$

eq. (34) and eq. (37) can be combined obtaining [153]

$$
\begin{aligned}
& \frac{D^{2} N^{\delta}}{d \tau^{2}}=R_{\alpha \beta \gamma}{ }^{\delta} u^{\alpha} u^{\gamma} N^{\beta}+\left(R_{\alpha \beta \gamma ; \varepsilon}^{\delta}-R_{\gamma \varepsilon \alpha ; \beta}^{\delta}\right) u^{\alpha} u^{\beta} N^{\gamma} N^{\varepsilon} \\
& +2 R_{\alpha \beta \gamma}^{\delta} u^{\beta} \frac{D N^{\alpha}}{d \tau} N^{\gamma}+\mathrm{O}\left(r_{0}^{3}\right) .
\end{aligned}
$$

Thus, it is possible writing the expansion of $x^{\alpha}\left(\tau, r_{0}\right)$ in terms of $N^{\alpha}$ [153]

$$
x^{\alpha}\left(\tau, r_{0}\right)=x^{\alpha}(\tau, 0)+N^{\alpha}-\Gamma_{\beta \gamma}^{\alpha} N^{\beta} N^{\gamma}+\mathrm{O}\left(r_{0}^{3}\right) .
$$

This formula shows that in the frame of the local observer (in which it is $\Gamma_{\beta \gamma}^{\alpha}=0$ along the central geodesic line [153]) the spatial components of $N^{\alpha}$ will directly give the time-dependent position of the nearby test mass. According to eq. (40), these positions include the next-order corrections, as compared with solutions to eq. (34).

Now, let us specialize to the scalar GW metric eq. (29) . We take into account only the linear perturbations in terms 
of the scalar GW amplitude $\Phi$. The first test mass is described by the central timelike geodesic $x^{i}(t)=0$. The correspondent tangent vector is $u^{\alpha}=(1,0,0,0)$. The second test mass is situated in the unperturbed position $x^{i}(0)=l^{i}$ having zero unperturbed velocity [153]. We assume that the frame of the local observer is located along the central geodesic. The goal is to find the trajectory of the second test mass using the geodesic deviation equation eq. (40). The deviation vector can be written like [153]

$$
N^{i}(t)=l^{i}+\delta l^{i}(t)
$$

where the variation in distance $\delta l^{i}(t)$ is caused by the scalar GW. Using the frame of the local observer, one can replace all the covariant derivatives in eq. (40) by ordinary derivatives $[153,154]$. In the lowest approximation eq. (40) reduces to eq. (34) and specializes to

$$
\frac{d^{2} \delta l^{i}(t)}{d t^{2}}=-\frac{1}{2} l^{j} \frac{\partial^{2}}{\delta t^{2}} \Phi \delta_{j}^{i}=\frac{1}{2} \omega^{2} l^{j} \Phi e_{j}^{(s) i}
$$

in the field of a scalar GW eq. (29). The relevant solution to eq. (43) coincides exactly with the usual electric part of the motion given by equation eq. (8). As we want to identify the magnetic part of the gravitational force arising from a scalar $\mathrm{GW}$, all the terms in eq. (40) have to be considered. Since $\frac{D N^{a}}{d \tau}$ is of the order of $\Phi$, the third term of eq. (40) is of the order of $\Phi^{2}$ and can be neglected. Working out the derivatives of the curvature tensor and substituting them into equation eq. (40) specialized in the field of a scalar GW eq. (29) , the accurate equations of motions read

$$
\frac{d^{2} \delta l^{i}(t)}{d t^{2}}=\frac{1}{2} \omega^{2} l^{j} \Phi e_{j}^{(s) i}-\frac{1}{2} \omega^{2} l^{k} l^{l}\left(k_{l} \delta^{i j}+\frac{1}{2} k^{i}\right) \delta_{l}^{j} \Phi e_{k j}^{(s)} .
$$

In this equation, which clearly exhibits two contributions, the second term is responsible for the magnetic component of motion and can be interpreted as the gravitational analog of the magnetic part of the Lorentz force (see also the analogy for ordinary tensor waves in previous Subsection).

\subsection{Variation of Distances Between Test Masses and Response of Interferometers for the Scalar magnetic Component}

It was already recalled that the previous descriptions in the frame of the local observer are as close as possible to the description of laboratory physics. As all the questions concerning test masses positions have been analysed, now it is possible discussing the variation of distances. We are interested in the distance between the central particle, located at coordinate origin, and the particle located, on average, at some position $\left(l_{1}, l_{2}, l_{3}\right)$. This model represents the situation of the beam - splitter and one mirror of an interferometer $[153,160]$. In the frame of the local observer the line element is given by equation [154]

$$
d s^{2}=-\left(d x^{0}\right)^{2}+\delta_{i j} d x^{i} d x^{j}+\mathrm{O}\left(\left|x^{j}\right|^{2}\right) d x^{\alpha} d x^{\beta} ;
$$

which gives the Galileian distance

$$
d(t)=\sqrt{x^{2}+y^{2}+z^{2}}+\mathrm{O}\left([\Phi l(\omega l)]^{2}\right) .
$$

Eq. (46) is accurate for terms of the order of $\Phi l$ and $\Phi l^{2} \omega$ inclusive, while the terms quadratic in $\Phi$ are neglected. Putting

$$
\begin{aligned}
& x=l_{1}+\delta x \\
& y=l_{2}+\delta y \\
& z=l_{3}+\delta z,
\end{aligned}
$$

we get [153]

$$
d(t)=l+\frac{1}{l}\left(l_{1} \delta x+l_{2} \delta y+l_{3} \delta z\right)
$$

and, using the time dependent positions eq. (33), the distance $d(t)$ is obtained with the required approximation (i.e. $\omega l=1$ )

$d(t)=l+\frac{1}{2 l}\left(l_{1}^{2}-l_{2}^{2}\right) \Phi(\omega t)-\frac{1}{4 l} \omega l_{3}\left(l_{1}^{2}-l_{2}^{2}\right) \Phi\left(\omega t-\frac{\pi}{2}\right)$.

Clearly, the first correction to $l$ is due to the electric contribution, while the second correction to $l$ is due to the magnetic contribution.

Now, let us compute the response of a laser interferometer. To compute the response function for an arbitrary propagating direction of the scalar $\mathrm{GW}$ one recalls that the arms of the interferometer are in the $\bar{u}$ and $\bar{v}$ directions, while the $x, y, z$ frame is adapted to the propagating scalar GW. Then, once again, the spatial rotation of the coordinate eq. (12) has to be performed.

In this way the scalar $\mathrm{GW}$ is propagating from an arbitrary direction $r$ to the interferometer (see Fig. 2).

At this point, one recalls that the response function is given by

$$
\delta d(t) \equiv d_{u}(t)-d_{v}(t)
$$

where $d_{u}(t)$ and $d_{v}(t)$ are the distances in the $u$ and $v$ directions, and, using equations eq. (49), eq. (12), and eq. (50) it is

$$
\begin{aligned}
& \delta d(t)=-\Phi(t) l \sin ^{2} \theta \cos 2 \phi+\Phi(t) \omega l^{2} \frac{1}{4} \cos \theta \\
& \left\{\left[\left(\frac{1+\sin ^{2} \theta}{2}\right)+\sin ^{2} \theta \sin ^{2} \phi\right](\cos \phi-\sin \phi)\right\} .
\end{aligned}
$$

In this equation the first term is due to the electric contribution, while the second term is due to the magnetic contribution [153]. The function

$$
\omega l \frac{1}{4} \cos \theta\left\{\left[\left(\frac{1+\sin ^{2} \theta}{2}\right)+\sin ^{2} \theta \sin ^{2} \phi\right](\cos \phi-\sin \phi)\right\}
$$

represents the so-called angular pattern [153] of interferometers for the magnetic contribution. The 
frequency-dependence in this angular pattern renders the magnetic component important in the high-frequency portion of the interferometers sensitivity band. Its value is shown in Fig. (5) for the LIGO interferometer for the frequency $f=8000 \mathrm{~Hz}$, which falls in such a high-frequency portion.

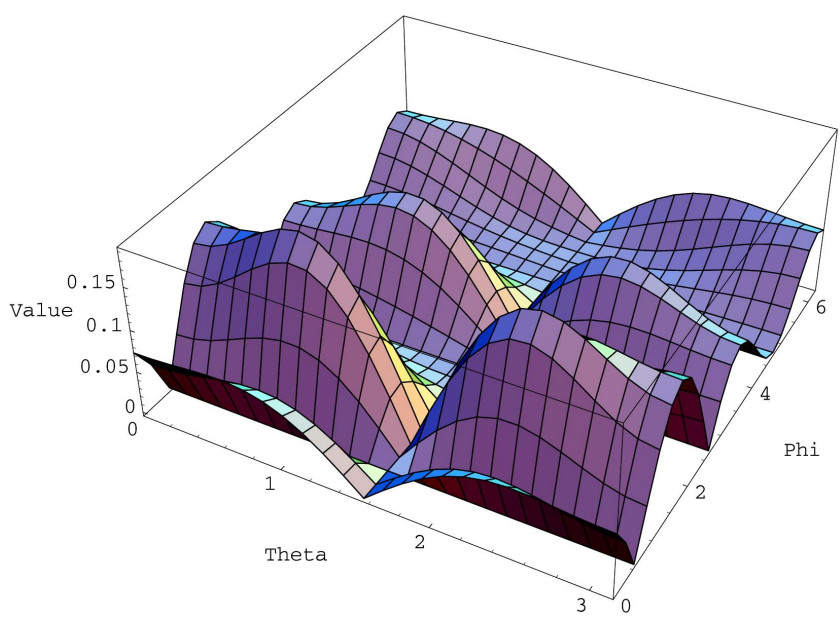

Fig. (5). the angular dependence of the total response function of the LIGO interferometer to the magnetic component of a SGW for $f=8000 \mathrm{~Hz}$.

Again, from the Figure, it looks clear that if one neglects the magnetic contribution, approximately $15 \%$ of currently observable signal could, in principle, be lost [153]. Finally, we recall that gravitomagnetic effects are important in the framework of the production of GWs from astrophysical sources too. This point has been emphasized in two recent papers $[161,162]$.

\section{CONCLUSION REMARKS}

After extensively reviewing general relativistic gravitomagnetism, both historically and phenomenologically, the so-called magnetic components of GWs have been reviewed in detail. Such components have to be taken into account in the context of the total response functions of interferometers for GWs propagating from arbitrary directions. Following the more recent approaches of this important issue, the analysis of such magnetic components has been reviewed in both of standard GTR and Scalar Tensor Gravity. Thus, it has been shown in detail that such a magnetic component becomes particularly important in the high-frequency portion of the range of ground based interferometers for GWs which arises from the two different theories of gravity. the reviewed results have shown that if one neglects the magnetic contribution to the gravitational field of a $\mathrm{GW}$, approximately $15 \%$ of the potential observable signal could, in principle, be lost.

\section{REFERENCES}

[1] Thorne KS. Gravitomagnetism, jets in quasars, and the stanford gyroscope experiment. In: Fairbank JD, Deaver BS, Everitt CWF, Michelson PF, Eds. Near Zero: New Frontiers of Physics. W. H. Freeman and Company: New York 1988; pp. 573-86.

[2] Rindler W. Relativity. special, general and cosmological. Oxford University Press: Oxford 2001.

[3] Mashhoon B. Gravitoelectromagnetism: a brief review. In: Iorio L, Ed. The measurement of gravitomagnetism: a challenging enterprise. Nova: Hauppauge 2007; pp. 29-39.
[4] Ruggiero ML, Tartaglia A. Gravitomagnetic effects. Il Nuovo Cimento B 2002; 117: 743-68.

[5] Schäfer G. Gravitomagnetic effects. Gen Relat Gravity 2004; 36 : 2223-35.

[6] Einstein A. Die Grundlage der allgemeinen Relativitätstheorie. Annalen der Physik 1916; 354: 769-822.

[7] Einstein A. Die Feldgleichungen der Gravitation. Sitzungsberichte der Königlich Preußischen Akademie der Wissenschaften 1915: 844-7.

[8] Ohanian HC, Ruffini RJ. Gravitation and Spacetime. 2nd ed. W.W. Norton \& Company: New York 1994.

[9] Maxwell JC. A Treatise on Electricity and Magnetism. Oxford: Clarendon Press 1873.

[10] Mashhoon B. Iorio L, Lichtenegger H. On the gravitomagnetic clock effect. Phys Lett A 2001; 292: 49-57.

[11] Coulomb CA. Premier Mémoire sur l'Electricité et le Magnétisme. Histoire de l'Académie Royale des Sciences 1785: 569-77.

[12] Coulomb CA. Sécond Mémoire sur l'Electricité et le Magnétisme. Histoire de l'Académie Royale des Sciences 1785: 578-611.

[13] Coulomb CA. Troisième Mémoire sur l'Electricité et le Magnétisme. Histoire de l'Académie Royale des Sciences 1785: 612-38.

[14] Newton I. Philosophiæ Naturalis Principia Mathematica. London: Joseph Streater for the Royal Society 1687. Vol. 3.

[15] Ampère A-M. Mémoire sur la théorie mathématique de phénomènes électrodynamiques uniquement déduite de l'experience, dans lequel se trouvent réunis les Mémoires que M. Ampère a communiqués à l'Académie royale des Sciences, dans les séances des 4 et 26 décembre 1820, 10 juin 1822, 22 decembre 1823, 12 septembre et 21 novembre 1825. Mémoires de l'Académie royale des Sciences de l'Institut de France, Année 1823, Tome VI. Firmin Didot: Paris 1827; pp. 175-388.

[16] Holzmüller G. Ueber die Anwendung der Jacobi-Hamilton'schen Methode auf den Fall der Anziehung nach dem elektrodynamischen Gesetze von Weber. Zeitschrift für Mathematik und Physik 1870; 15: 69-91.

[17] Weber W. Elektrodynamische Massbestimmungen über ein allgemeines Grundgesetz der elektrischen Wirkung. Abhandlungen der Königlichen Sächsischen Gesellschaft der Wissenshaften 1846: 211-378.

[18] Weber W. Elektrodynamische Massbestimmungen. Annalen der Physik und Chemie 1848; 73: 193-240.

[19] Tisserand FF. Sur le mouvement des planètes au tour du Soleil, d'après la loi électrodynamique de Weber. Comptes Rendus de l' Académie des Sciences (Paris) 1872; 75: 760-3.

[20] Tisserand FF. Sur le mouvement des planètes, en supposant l'attraction représentée par l'une des lois électrodynamiques de Gauss ou de Weber. Comptes Rendus de l' Académie des Sciences (Paris) 1890; 100: 313-5.

[21] Einstein A. Erklärung der Perihelbewegung des Merkur aus der allgemeinen Relativitätstheorie. Sitzungsberichte der Preußischen Akademie der Wissenschaften 1915; 2: 831-9.

[22] Le Verrier U. Lettre de M. Le Verrier à M. Faye sur la Théorie de Mercure et sur le Mouvement du Périhélie de cette Planète. Comptes Rendus de l' Académie des Sciences (Paris) 1859; 49: 379-83.

[23] Lévy M. Sur l'application des lois électrodynamiques au mouvement des planètes. Comptes Rendus de l' Académie des Sciences (Paris) 1890; 110: 545-51.

[24] Maxwell JC. A Dynamical Theory of the Electromagnetic Field. Phil Trans R Soc Lond 1865; 155: 459-512.

[25] Assis AKT. Deriving Gravitation from Electromagnetism. Can J Phys 1992; 70: 320-40.

[26] Heaviside O. Electromagnetic Theory, Vol. 1. The Electrician: London 1894; pp. 455-65.

[27] Mach E. Die Geschichte und die Wurzel des Satzes von der Erhaltung der Arbeit. Calve: Prag 1872.

[28] Mach E. Die Mechanik in ihrer Entwicklung. Historisch-kritisch dargestellt. Broackhaus: Leipzig 1883.

[29] Friedländer B., Friedländer I. Absolute oder relative Bewegung? Leonhard Simion Verlag: Berlin 1896.

[30] Sciama DW. On the origin of inertia. Mon Not R Astron Soc 1953; 113: 34-42.

[31] Einstein A. Zur Elektrodynamik bewegter Körper. Annalen der Physik 1905; 322: 891-921. 
[32] Einstein A. Zum gegenwärtigen stande des Gravitationsproblem. Physikalische Zeitschrift 1913; 14: 1249-66.

[33] Khan AR, O'Connell RF. Gravitational analog of magnetic force. Nature 1976; 261: 480-1.

[34] Bedford D, Krumm P. On relativistic gravitation. Am J Phys 1985; 53: 889-90.

[35] Kolbenstvedt H. Gravomagnetism in special relativity. Am J Phys 1988; 56: 523-4.

[36] Klein MJ, Kox AJ, Schulmann R, Eds. The Collected Papers of Albert Einstein. Vol. 4. The Swiss Years: Writings, Princeton: 1912-1914. Princeton University Press, 1995; pp. 344-473.

[37] Einstein A. Zur allgemeinen Relativitätstheorie. Sitzungsberichte der Königlich Preußischen Akademie der Wissenschaften 1915: 778-86.

[38] de Sitter W. Einstein's theory of gravitation and its astronomical consequences. Mon Not R Astron Soc 1916; 76: 699-728.

[39] Thirring H. Über die formale Analogie zwischen den elektromagnetischen Grundgleichungen und den Einsteinschen Gravitationsgleichungen erster Näherung. Physikalische Zeitschrift 1918; 19: 204-5.

[40] Thirring H. Über die Wirkung rotierender ferner Massen in der Einsteinschen Gravitationstheorie. Physikalische Zeitschrift 1918; 19: 33-9

[41] Thirring H. Berichtigung zu meiner Arbeit: "Über die Wirkung rotierender ferner Massen in der Einsteinschen Gravitationstheorie". Physikalische Zeitschrift 1921; 22: 29-30.

[42] Schulmann R, Kox AJ, Janssen M, Illy J, Eds. The Collected Papers of Albert Einstein. Vol. 8. The Berlin Years: Correspondence, 1914-1918. Princeton: Princeton University Press 1998; Documents 361, 369, 401, 405.

[43] Pfister H. On the history of the so-called Lense-Thirring effect. Gen Relat Gravit 2007; 39: 1735-48.

[44] Lense J., Thirring H. Über den Einfluß der Eigenrotation der Zentralkörper auf die Bewegung der Planeten und Monde nach der Einsteinschen Gravitationstheorie. Physikalische Zeitschrift 1918; 19: 156-63.

[45] Pugh GE. Proposal for a satellite test of the Coriolis prediction of general relativity. WSEG Research Memorandum No. 11. Washington DC: The Pentagon 1959.

[46] Schiff LI. Possible new experimental test of general relativity theory. Phys Rev Lett 1960; 4: 215-7.

[47] Schiff LI. On experimental tests of the general theory of relativity. Am J Phys 1960; 28: 340-3.

[48] Schiff LI. Motion of gyroscope according to Einsteinís theory of gravitation. Proc Natl Acad Sci USA 1960; 46: 871-82.

[49] Rindler W. The Lense-Thirring effect exposed as anti-Machian. Phys Lett A 1994; 187: 236-8.

[50] Bondi H., Samuel J. The Lense-Thirring effect and Mach's principle. Phys Lett A 1997; 228: 121-6.

[51] Schmid C. Cosmological gravitomagnetism and Mach's principle. Phys Rev D 2006; 74: 044031.

[52] Schmid C. Mach's principle: Exact frame-dragging via gravitomagnetism in perturbed Friedmann-Robertson-Walker universes with $K=( \pm 1,0)$. Phys Rev D 2009; 79: 064007 .

[53] Pfister H., Braun KH. Induction of correct centrifugal force in a rotating mass shell. Class Quantum Gravity 1985; 2: 909-18.

[54] Matte A. Sur de nouvelles solutions oscillatoires des equations de la gravitation. Can J Math 1953; 5: 1-16.

[55] Costa LFO, Herdeiro CAR. Gravitoelectromagnetic analogy based on tidal tensors. Phys Rev D 2008; 78: 024021.

[56] Pascual-Sánchez J-F. On the (Non) Existence of a Gravitomagnetic Dynamo. In: Ruffini RJ, Sigismondi C, Eds. Nonlinear Gravitodynamics. The Lense-Thirring Effect. Singapore: World Scientific 2003; pp. 128-34.

[57] Tartaglia A, Ruggiero ML. Analogies and differences between gravito-electromagnetism and electromagnetism. In: Iorio L, Ed. The measurement of gravitomagnetism: a challenging enterprise. Hauppauge: Nova 2007; pp. 41-50.

[58] Pascual-Sánchez J-F. On the (Non) Existence of Several Gravitomagnetic Effects. In: Molina A, Martn J, Ruiz E, Atrio F, Eds. Gravitation and relativity in general. Singapore: World Scientific 1999; pp. 291-6.

[59] Föppl A. Über einen Kreiselversuch zur Messung der Umdrehungsgeschwindigkeit der Erde. Sitzungsberichte der Bayerischen Akademie der Wissenschaften 1904; 34: 5-28.
[60] Ginzburg VL. The use of artificial earth satellites for verifying the general theory of relativity. Uspekhi Fizicheskikh Nauk (Advances in Physical Science) 1957; 63: 119-22.

[61] Ginzburg VL. Artificial satellites and the theory of relativity. Sci Am 1959; 200: 149-60.

[62] Ginzburg VL. Experimental verifications of the general theory of relativity. In: recent developments in general relativity. London: Pergamon Press 1962; pp. 57-71.

[63] Bogorodskii AF. Relativistic effects in the motion of an artificial earth satellite. Sov Astron 1959; 3; 857-62.

[64] Yilmaz H. Proposed Test of the Nature of Gravitational Interaction. Bull Am Phys Soc 1959; 4. 65.

[65] van Patten RA, Everitt CWF. Possible experiment with two counter-orbiting drag-free satellites to obtain a new test of Einstein's general theory of relativity and improved measurements in geodesy. Phys Rev Lett 1976; 36: 629-32.

[66] van Patten RA, Everitt CWF. A possible experiment with two counter-orbiting drag-free satellites to obtain a new test of einstein's general theory of relativity and improved measurements in geodesy. Celestial Mech Dyn Astron 1976; 13: 429-47.

[67] Cugusi L, Proverbio E. Relativistic effects on the motion of the earth's. Satellites, paper presented at the International Symposium on Satellite Geodesy in Budapest from June 28 to July 1, 1977. J Geodesy 1977; 51: 249-52.

[68] Cugusi L, Proverbio E. Relativistic effects on the motion of earth's artificial satellites. Astron Astrophys 1978; 69: 321-25.

[69] Ciufolini I. Measurement of the Lense-Thirring drag on highaltitude, laser-ranged artificial satellites. Phys Rev Lett 1986; 56: 278-81.

[70] Iorio L. A new proposal for measuring the Lense-Thirring effect with a pair of supplementary satellites in the gravitational field of the Earth. Phys Lett A 2003; 308: 81-4.

[71] Ciufolini I, Lucchesi DM, Vespe F, Mandiello A. Measurement of dragging of inertial frames and gravitomagnetic field using laserranged satellites. Il Nuovo Cimento A 1996; 109: 575-90.

[72] Ciufolini I. On a new method to measure the gravitomagnetic field using two orbiting satellites. Il Nuovo Cimento A 1996; 109: 170920.

[73] Ciufolini I, Pavlis EC. A confirmation of the general relativistic prediction of the Lense-Thirring effect. Nature 2004; 431: 958-60.

[74] Ciufolini I, Pavlis EC, Peron R. Determination of frame-dragging using Earth gravity models from CHAMP and GRACE. New Astronomy 2006; 11: 527-50.

[75] Lucchesi DM. The Lense Thirring effect measurement and LAGEOS satellites orbit analysis with the new gravity field model from the CHAMP mission. Adv Space Res 2007; 39: 324-32.

[76] Ries JC, Eanes RJ, Watkins MM. Confirming the frame-dragging effect with satellite laser ranging. In: Schillak S, Ed. Proceedings of The 16th International Laser Ranging Workshop. "SLR-The Next Generation", Poznan' (PL), 13-17 October 2008. Available from: http://cddis.gsfc.nasa.gov/lw16/

[77] Iorio L. The new Earth gravity models and the measurement of the Lense-Thirring effect. In: Novello M, Bergliaffa SP, Ruffini R, Eds. The tenth marcel grossmann meeting on recent developments in theoretical and experimental general relativity, gravitation and relativistic field theories. Proceedings of the MG10 Meeting, Rio de Janeiro, Brazil 20-26 July 2003. World Scientific: Singapore 2006; pp. 1011-20.

[78] Iorio L. On the reliability of the so-far performed tests for measuring the Lense-Thirring effect with the LAGEOS satellites. N Astron 2005; 10: 603-15.

[79] Ciufolini I, Pavlis EC. On the measurement of the LenseñThirring effect using the nodes of the LAGEOS satellites, in reply to "On the reliability of the so-far performed tests for measuring the Lense-Thirring effect with the LAGEOS satellites" by L. Iorio. N Astron 2005; 10: 636-51.

[80] Lucchesi DM. The impact of the even zonal harmonics secular variations on the Lense-Thirring effect measurement with the two lageos satellites. Int J Mod Phys D 2005; 14: 1989-2023.

[81] Iorio L. A Critical Analysis of a Recent Test of the Lense-Thirring Effect with the LAGEOS Satellites. J Geod 2006; 80: 128-36.

[82] Iorio L. An assessment of the measurement of the Lense-Thirring effect in the Earth gravity field, in reply to: "On the measurement of the Lense-Thirring effect using the nodes of the LAGEOS satellites, in reply to "On the reliability of the sofar performed tests for measuring the Lense-Thirring effect with the LAGEOS 
satellites" by L. Iorio," by I. Ciufolini and E. Pavlis. Planet Space Sci 2007; 55: 503-11.

[83] Iorio L. An Assessment of the Systematic Uncertainty in Present and Future Tests of the Lense-Thirring Effect with Satellite Laser Ranging. Space Sci Rev 2009; 148: 363-81.

[84] Iorio L. Conservative evaluation of the uncertainty in the LAGEOS-LAGEOS II Lense-Thirring test. Central Eur J Phys 2010; 8: 25-32.

[85] Casotto S, Ciufolini I, Vespe F, Bianco G. Earth satellites and gravitomagnetic field. Il Nuovo Cimento B 1990; 105: 589-99.

[86] Iorio L. Is it possible to improve the present LAGEOS-LAGEOS II Lense-Thirring experiment? Class Quantum Gravity 2002; 19: 5473-80.

[87] Iorio L, Doornbos E. How to reach a few percent level in determining the Lense-Thirring effect? Gen Relat Gravitation 2005; 37: 1059-74.

[88] Vespe F, Rutigliano P. The improvement of the Earth gravity field estimation and its benefits in the atmosphere and fundamental physics. Adv Space Res 2005; 36: 472-85.

[89] Iorio L. On the use of Ajisai and Jason-1 satellites for tests of general relativity. N Astron 2006; 12: 224-33.

[90] Iorio L. The impact of the new Earth gravity models on the measurement of the Lense-Thirring effect with a new satellite. $\mathrm{N}$ Astron 2005; 10: 616-35.

[91] Ciufolini I, Pavlis EC. Measurement of gravitomagnetism with satellite laser ranging to LAGEOS, LAGEOS 2 and LARES satellites. Bull Am Astron Soc 2009; 41: 890.

[92] Iorio L. A critical approach to the concept of a polar, low-altitude LARES satellite. Class Quantum Gravity 2002; 19: L175-83.

[93] Iorio L. A comment on the paper "On the orbit of the LARES satellite”, by I. Ciufolini. Planet Space Sci 2007; 55: 1198-200.

[94] Iorio L. Towards a 1\% measurement of the Lense-Thirring effect with LARES? Adv Space Res 2009; 43: 1148-57.

[95] Iorio L. Will the LARES mission be able to measure the LenseThirring effect at 1\%? Gen Relat Gravity 2009; 41: 1717-24.

[96] Iorio L. A note on the evidence of the gravitomagnetic field of Mars. Class Quantum Gravity 2006; 23: 5451-4.

[97] Iorio L. Is it possible to measure the Lense-Thirring effect in the gravitational fields of the sun and of mars? In: Iorio L, Ed. The measurement of gravitomagnetism: a challenging enterprise. Nova: Hauppauge 2007; pp. 177-188.

[98] Krogh K. Comment on 'Evidence of the gravitomagnetic field of Mars'. Class Quantum Gravity 2007; 24: 5709-15.

[99] Iorio L. On the Lense-Thirring test with the Mars Global Surveyor in the gravitational field of Mars. Cent Eur J Phys 2010; 8: 509513 .

[100] Iorio L. Mars and frame-dragging: study for a dedicated mission. Gen Relat Gravity 2009; 41: 1273-84.

[101] Iorio L, Lainey V. The Lense-Thirring effect in the Jovian system of the Galilean satellites and its measurability. Int J Mod Phys D 2005; 14: 2039-49.

[102] Pitjeva EV. Relativistic effects and solar oblateness from radar observations of planets and spacecraft. Astron Lett 2005; 31: 3409.

[103] Pitjeva EV. Ephemerides EPM2008: the updated model, constants, data. In: Soffel M., Capitaine N., Eds. Proceedings of the "Journées 2008 Systèmes de référence spatio-temporels". Lohrmann-Observatorium and Observatoire de Paris 2009: pp. 5760.

[104] Iorio L. Is it possible to measure the Lense-Thirring effect on the orbits of the planets in the gravitational field of the Sun? Astron Astrophys 2005; 431: 385-9.

[105] Iorio L. First preliminary tests of the general relativistic gravitomagnetic field of the Sun and new constraints on a Yukawalike fifth force from planetary data. Planet Space Sci 2007; 55: 1290-8.

[106] Iorio L. Advances in the measurement of the Lense-Thirring effect with planetary motions in the field of the Sun. Sch Res Exch 2008; 2008: 105235.

[107] Ni WT. ASTROD and ASTROD I-Overview and Progress. Int J Mod Phys D 2008; 17: 921-40.

[108] Fairbank WM, Schiff LI. Proposed experimental test of general relativity. Proposal to NASA. Stanford University: Stanford 1961.

[109] Everitt CWF. The gyroscope experiment I. General description and analysis of gyroscope performance. In: Bertotti B, Ed. Experimental Gravitation: Proceedings of Course 56 of the
International School of Physics "Enrico Fermi". New York: Academic Press 1974; pp. 331-60.

[110] Everitt CWF, Buchman S, DeBra DB, et al. Gravity Probe B: Countdown to launch. In: Lämmerzahl C, Everitt CWF, Hehl FW, Eds. Gyros, Clocks, Interferometers. Testing Relativistic Gravity in Space, Berlin: Springer Verlag 2001; pp. 52-82.

[111] Conklin JW. The Gravity Probe B experiment and early results. J Phys Conf Ser 2008; 140: 012001.

[112] Everitt CWF, Adams M, Bencze W, et al. Gravity Probe B Data Analysis. Space Sci Rev 2009; 148: 53-69.

[113] Muhlfelder B, Adams M, Clarke B, et al. GP-B Systematic error determination. Space Sci Rev 2009; 148: 429-39.

[114] Keiser GM, Kolodziejczak J, Silbergleit AS. Misalignment and resonance torques and their treatment in the GP-B data analysis. Space Sci Rev 2009; 148: 383-95.

[115] Silbergleit AS, Conklin J, DeBra D, et al. Polhode Motion, trapped flux, and the GP-B science data analysis. Space Sci Rev 2009; 148: 397-409.

[116] Haas MR, Ross DK. Measurement of the angular momentum of Jupiter and the Sun by use of the Lense- Thirring effect. Astrophys Space Sci 1975; 32: 3-11.

[117] Nordtvedt K. Existence of the gravitomagnetic interaction. Int J Theor Phys 1988; 27: 1395-404.

[118] Nordtvedt K. Some considerations on the varieties of frame dragging. In: Ruffini RJ, Sigismondi C, Eds. Nonlinear gravitodynamics: the Lense-Thirring effect. Singapore: World Scientific 2003; pp. 35-45.

[119] Murphy TW, Nordtvedt K, Turyshev SG. Gravitomagnetic influence on Gyroscopes and on the Lunar Orbit. Phys Rev Lett 2007; 98: 071102 .

[120] Kopeikin SM. Comment on "Gravitomagnetic influence on Gyroscopes and on the Lunar Orbit”. Phys Rev Lett 2007; 98: 229001.

[121] Murphy TW, Nordtvedt K, Turyshev SG. Murphy, Nordtvedt, and Turyshev Reply. Phys Rev Lett 2007; 98: 229002.

[122] Soffel M, Klioner S, Müller J, Biskupek L. Gravitomagnetism and lunar laser ranging. Phys Rev D 2008; 78: 024033.

[123] Ciufolini I. Frame-dragging, gravitomagnetism and Lunar Laser Ranging. N Astron 2010; 15: 332-7.

[124] Iorio L. Will it be possible to measure intrinsic gravitomagnetism with lunar laser ranging? Int J Mod Phys D 2009; 18: 1319-26.

[125] Fomalont EB, Kopeikin SM. Radio interferometric tests of general relativity. In: Jin WJ, Platais I, Perryman MAC, Eds. A Giant Step: From Milli- to Micro-Arcsecond Astrometry: Proceedings IAU Symposium No. 248, 2007. Cambridge: Cambridge University Press 2008; p. 383.

[126] Iorio L, Ed. The Measurement of Gravitomagnetism: A Challenging Enterprise. Nova: Hauppauge 2007.

[127] Thorne KS, Price RH, Macdonald DM, Eds. Black holes: the membrane paradigm: New Haven Yale University Press 1986.

[128] Ruffini RJ, Sigismondi C, Eds. Nonlinear gravitodynamics: the Lense-Thirring effect. Singapore: World Scientific 2003; pp. 235345.

[129] Stella L, Possenti A. Lense-Thirring precession in the astrophysical context. Space Sci Rev 2009; 148: 105-21.

[130] Giazotto A. Status of gravitational wave detection. J Phys Conf Ser 2008; 120: 032002.

[131] Hulse RA, Taylor JH. Discovery of a pulsar in a binary system. Astrophys J Lett 1975; 195 L51-3.

[132] Corda C. Interferometric detection of gravitational waves: the definitive test for General Relativity. Int J Mod Phys D 2009; 18 : 2275-82.

[133] Einstein A. Zur allgemeinen Relativitätstheorie. Sitzungsberichte der Königlich Preußischen Akademie der Wissenschaften 1915: 778-86.

[134] Einstein A. Näherungsweise Integration der Feldgleichungen der Gravitation. Sitzungsberichte der Königlich Preußischen Akademie der Wissenschaften 1916: 688-96.

[135] Einstein A. Über Gravitationswellen. Sitzungsberichte der Königlich Preußischen Akademie der Wissenschaften 1918: 154-67

[136] Smoot GF, Steinhardt PJ. Gravity's rainbow. Gen Relat Gravity 1993; 25: 1095-100

[137] Grishchuk LP. Amplification of gravitational waves in an isotropic universe. J Exp Theor Phys 1975; 40: 409-15.

[138] Starobinskii AA. Spectrum of relic gravitational radiation and the early state of the universe. J Exp Theor Phys Lett 1979; 30: 682-5. 
[139] Allen B. The stochastic gravity-wave background: sources and detection. In: Marck J-A, Lasota J-P, Eds. Relativistic gravitation and gravitational radiation: Cambridge: Cambridge University Press 1997; pp. 373-417.

[140] Corda C. Primordial production of massive relic gravitational waves from a weak modification of general relativity. Astropart Phys 2008; 30: 209-15.

[141] Lyth DH, Liddle AR. Primordial density perturbation. Cambridge: Cambridge University Press 2009.

[142] Spergel DN, Verde L, Peiris HV, et al. First year wilkinson microwave anisotropy probe (WMAP) observations: determination of cosmological parameters. Astrophys J Suppl 2003; 148: 175-94.

[143] Corda C. A review of the stochastic background of gravitational waves in $f(R)$ gravity with WMAP constrains. Open Astron J. Available from http://arxiv.org/abs/0901.1193

[144] Pirani FAE. Invariant formulation of gravitational radiation theory. Phys Rev 1957; 105: 1089-99.

[145] Weber J. Gravitational waves. First Award at the 1959 Gravity Research Foundation Competion. Available from http://www.gravityresearchfoundation.org

[146] Weber J. Detection and generation of gravitational waves. Phys Rev 1960; 117: 306-13.

[147] Weber J. Evidence for discovery of gravitational radiation. Phys Rev Lett 1969; 22: 1320-4.

[148] Corda C. The Virgo-MiniGRAIL cross correlation for the detection of scalar gravitational waves. Mod Phys Lett A 2007; 22: 2647-55.

[149] Baskaran D, Grishchuk LP. Components of the gravitational force in the field of a gravitational wave. Class Quantum Gravity 2004; 21: 4041-61.

[150] Corda C. The importance of the "magnetic" components of gravitational waves in the response functions of interferometers. Int J Mod Phys D 2007; 16: 1497-517.

[151] Corda C. "Extension of the frequency-range of interferometers for the "magnetic" components of gravitational waves?". In:
Dumarchez J, Van JTT, Eds. Proceedings of the XLIInd Rencontres de Moriond, Gravitational Waves and Experimental Gravity. Hanoi: The Gioi Publishers 2007; pp. 95.

[152] Corda C. 'Magnetic' components of gravitational waves and response functions of interferometers. In: Halsey D, Raynor W, Eds. Handbook of Interferometers; Research, Technology and Applications. Hauppauge: Nova 2009; pp. 23-53.

[153] Corda C, Ali SA, Cafaro C. Interferometer response to scalar gravitational waves. Int J Mod Phys D 2010; 19: 2095-2109.

[154] Misner CW, Thorne KS, Wheeler JA. Gravitation. San Francisco: W.H.Feeman and Company 1973.

[155] Landau LD, Lifshitz EM. The classical theory of fields: Volume 2. Butterworth-Heinemann: Oxford 1980.

[156] Lee DL. Conservation laws, gravitational waves, and mass losses in the Dicke-Brans-Jordan theory of gravity. Phys Rev D 1974; 10: 2374-83.

[157] Damour T, Esposito-Farese G. Tensor-multi-scalar theories of gravitation. Class Quantum Gravity 1992; 9: 2093-176.

[158] Tobar ME, Suzuki T, Kuroda K. Detecting free-mass commonmode motion induced by incident gravitational waves. Phys Rev D 1999; 59: 102002.

[159] Nakao K, Harada T, Shibata M, Kawamura S, Nakamura T. Response of interferometric detectors to scalar gravitational waves. Phys Rev D 2001; 63: 082001.

[160] Capozziello S, Corda C. Scalar gravitational waves from scalartensor gravity: production and response of interferometers. Int $\mathbf{J}$ Mod Phys D 2006; 15: 1119-50.

[161] Capozziello S, De Laurentis M, Garufi F, Milano L. Relativistic orbit with gravitomagnetic effect. Phys Scr 2009; 79: 025901.

[162] Capozziello S, De Laurentis M, Forte L, Garufi F, Milano L. Relativistic orbit with gravitomagnetic effect. Phys Scr 2009; 79: 025901 .

Received: May 20, 2011

(C) Iorio and Corda; Licensee Bentham Open.

This is an open access article licensed under the terms of the Creative Commons Attribution Non-Commercial License (http://creativecommons.org/licenses/by-nc/3.0/) which permits unrestricted, non-commercial use, distribution and reproduction in any medium, provided the work is properly cited. 